\title{
QUASILINEAR ELLIPTIC PROBLEM WITH HARDY POTENTIAL AND A REACTION-ABSORBTION TERM
}

\author{
SOFIANE EL-HADI MIRI
}

Abstract. We consider the following quasilinear elliptic problem

$$
\left\{\begin{array}{cc}
-\Delta_{p} u \pm u^{q}=\lambda \frac{u^{p-1}}{|x|^{p}}+h & \text { in } \Omega, \\
u \geqslant 0 \text { and } u=0 & \text { on } \partial \Omega,
\end{array}\right.
$$

where, $1<p<N, \Omega \subset \mathbb{R}^{N}$ is a bounded regular domain such that $0 \in \Omega, q>p-1$ and $h$ is a nonnegative measurable function with suitable hypotheses. The main goal of this paper is to analyze the interaction between the Hardy potential, and the term $u^{q}$, in order to get existence and non existence of positive solution. We can summarize our main results, in the two following points:

(i) If $u^{q}$ appears as a reaction term, then we show the existence of a critical exponent $q_{+}(\lambda)$, such that for $q>q_{+}$, the considered problem has no positive distributional solution. If $q<q_{+}$ we find solutions under suitable hypothesis on $h$.

(ii) If $u^{q}$ appears as an absorption term, then there exists $q_{*}$ such that if $q>q_{*}$, the problem under consideration has a positive solution for all $\lambda>0$ and for all $h \in L^{1}(\Omega)$. The optimality of $q_{*}$ is proved in the sense that if $q<q_{*}$, then nonexistence holds if $\lambda>\Lambda_{N, p}$.

Mathematics subject classification (2010): 35K15, 35K55, 35K65, 35B05, 35B40.

Keywords and phrases: quasilinear elliptic problems, singular Hardy-Sobolev potential, comparison principle, existence and nonexistence results.

\section{REFERENCES}

[1] B. Abdellaoui, E. Collorado, I. Peral, Some improved Caffarelli-Kohn-Nirenberg inequalities, Calc. Var., 23 (2005), 327-345.

[2] B. Abdellaoui, V. Felli, I. Peral, Existence and nonexistence results for quasilinear elliptic equations involving the p-laplacian, Boll. Unione Mat. Ital. Sez. B. (8), 9, 2 (2006), 445-484.

[3] B. Abdellaoui, S.E. Miri, I. Peral, T.M. Touaoula, Some remarks on quasilinear parabolic problems with singular potential and a reaction term, to appear.

[4] B. AbDellaoui, I. Peral, Existence and nonexistence results for quasilinear elliptic equations involving the p-laplacian with a critical potential, Annal. Math. Pura. Appl., 182 (2003), 247-270.

[5] B. Abdellaoui, I. Peral, A note on a critical problem with natural growth in the gradient, Jour. Euro. Math. Soc, 6 (2006), 119-136.

[6] B. Abdelladoui, I. Peral, A. Primo, Breaking of resonance and regularizing effect of a first order term in some elliptic equations, Ann. I. H. Poincaré, 25 (2008), 969-985.

[7] B. Abdellaoui, A. Primo, T.M. Touaoula, Existence, nonexistence and multiplicity results for semilinear elliptic problems with measure data and absorption-reaction term, Differ. Equ. Appl., 1, 2 (2009), 253-284.

[8] W. Allegretto, Y. X. Huang, A Picone's identity for the p-Laplacian and applications, Nonlinear Analysis T. M. A., 32, 7 (1998), 819-830.

[9] A. Ambrosetti, P.H. Rabinowitz, Variational methods in critical point theory and applications, J. Functional Analysis, 14 (1973), 349-381. 
[10] P. Bénilan, L. Boccardo, T. Gallouët, M. Pierre, J.L. VÁzquez, An L L ${ }^{1}$-theory of existence and uniqueness of solutions of nonlinear elliptic equations, Ann. Scuola Norm. Sup. Pisa Cl. Sci. (4), 22, 2 (1995), 241-273.

[11] L. Boccardo, T. Gallouët, J.L. VazQuez, Nonlinear elliptic equations in $\mathbb{R}^{N}$ without growth restrictions on the data, J. Differential Equations, 105, 2 (1993), 334-363.

[12] H. BReZIS, L. Dupaigne, A. TeSEI, On a semilinear equation with inverse-square potential, Selecta Math., 11 (2005), 1-7.

[13] H. BRezis, S. KAMin, Sublinear elliptic equations in $\mathbb{R}^{N}$, Manuscripta Math, 74 (1992), 87-106.

[14] J. García Azorero, I. Peral, Hardy Inequalities and some critical elliptic and parabolic problems, J. Diff. Eq., 144 (1998), 441-476.

[15] M. PICONE, Sui valori eccezionali di un paramtro da cui dipende una equazione differenziale lineare ordinaria del secondo ordine, Ann. Scuola. Norm. Pisa., 11 (1910), 1-144.

[16] J.L. VÁZQUEZ, A strong maximum principle for some quasilinear elliptic equations, Applied Math. and Optimization, 12, 3 (1984), 191-202. 